from Dr. N.R. Pace. $Q \beta$ replicase and MNV11(+) RNA were supplied by Dr. C.K. Biebricher. We greatly acknowledge critical comments on the manuscript by Drs. M. Eigen and C.K. Biebricher and one of the referees. This work was in part supported through Grant No. 0310248A4 from the German BMBF and Grant No. Schw 578/11 from the DFG. Address correspondence to Dr. Andreas Schwienhorst, Department 081, Max-Planck-Institute for Biophysical Chemistry, Am Fassberg 2, 37077 Goettingen, Germany.

Received 27 May 1997; accepted 25 July 1997.

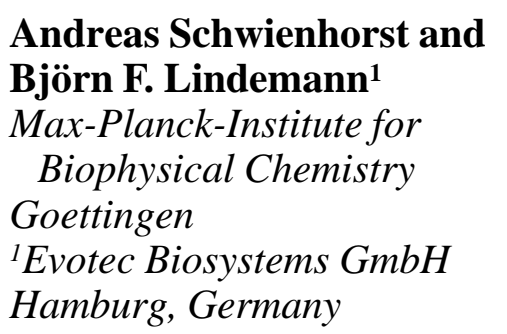

\section{Determination of Trans- gene Copy Number and Expression Level Using Denaturing Gradient Gel Electrophoresis}

BioTechniques 24:126-131 (January 1998)

\begin{abstract}
Transgenic mice and cell lines are frequently developed to study human disease. Accurate determination of transgene copy number and levels of mRNA are necessary to understand the phenotypic changes observed in these models. Currently, transgene copy number and expression are estimated by Southern blot analysis of genomic DNA and Northern blot analysis of $m R N A$. We report a novel PCR-based method for determining transgene copy number and levels of transgene expression using competitive PCR between endogenous genomic genes and mutant transgenes followed by denaturing
\end{abstract}

gradient gel electrophoresis (DGGE). We are able to accurately quantify a range of 1-10 copies of transgene incorporated per diploid genome. After reverse-transcribing RNA to cDNA, we are able to quantify levels of transgene $m R N A$ that correlate with biochemical and histological evidence of transgene activity. In conclusion, resolving PCR and reverse transcription- $P C R$ products by $D G G E$ is a rapid and reproducible method that allows for accurate determination of transgene copy number and expression. This technique provides a more complete understanding of transgene effects.

\section{INTRODUCTION}

Small nucleotide deletions, additions or substitutions can alter gene expression and may be associated with human diseases. One highly sensitive technique used to detect these nucleotide sequence changes is denaturing gradient gel electrophoresis (DGGE) $(1,4)$. In DGGE, DNA is electrophoresed through a polyacrylamide gel in which the concentrations of the denaturants urea and formamide increase linearly. As a double-stranded DNA fragment travels through the gel, it reaches a denaturant concentration that corresponds to its "melting point". At that point, a conformational change occurs that alters the mobility of the fragment. Denaturation occurs under characteristic conditions that reflect not only the length of a DNA fragment but also its sequence. To improve the ability of DGGE to detect small mutations throughout a DNA fragment, one polymerase chain reaction (PCR) primer is synthesized with a GC-rich sequence at its $5^{\prime}$ end to introduce a "GC clamp" with a high melting point into the DNA product. This transforms the amplified fragment into a single melting domain and improves the sensitivity of the technique (1). Thus DNA fragments that differ only by a single base can be distinguished by comparing their migration patterns through denaturing gradient gels $(1,4,7,9)$.

DGGE of a DNA sample that contains equal numbers of two DNA fragments that differ by only a single base, as occurs in many heterozygous genetic diseases, resolves four distinct bands. The two most rapidly migrating bands represent the two homoduplex DNA fragments, whereas the two more slowly migrating DNA fragments represent heteroduplex DNA fragments produced during PCR. Because the generation of homoduplex DNA fragments is based upon the competitive nature of the PCR, we hypothesized that DGGE could be used to determine the relative number of copies of two similar genes in a DNA sample. To test this hypothesis, we analyzed genomic DNA and cDNA from transgenic mice in which expression of a constitutively activated form of the alpha chain of the $G$ protein $\mathrm{Gq}(\mathrm{Q} 209 \mathrm{~L})$ that differs from wild-type murine $G_{\alpha q}$ by a single base pair (3) was targeted to the thyroid gland by linking Q209L to the rat thyroglobulin gene promoter (TGGQ) (8). In this paper, we describe the use of DGGE to determine the number of copies of the TGGQ transgene that have been integrated into the genome of the transgenic mice. In addition, by using DGGE to analyze cDNA prepared by reverse transcription (RT)-PCR of thyroid RNA from these mice, we have been able to compare levels of transgene-derived $\mathrm{Gq}$ mRNA to levels of genomic wild-type Gq mRNA.

\section{MATERIALS AND METHODS}

\section{Oligonucleotide Primers and Template DNA and RNA}

Oligonucleotide primers were designed to amplify a 149-bp region surrounding the Gln 209 codon of murine $\mathrm{G}_{\alpha q}(10)$. One primer of the pair ( $\mathrm{mdr}$ 2) was synthesized with a 40-base GCrich extension at the $5^{\prime}$ end (1). The sequence of the GC "clamp" is the following:

\section{5'-CGCCCGCCGCGCCCCGCGCC- CGCCCCGCCGCCCCCGCCCG-3'.}

The sequences of the primers are:

Sense: 5'-CAGAATGGTCGATGTAGGGGGC-3' (mdr 1)

Antisense: $5^{\prime}$-(GC) ${ }_{40}$ CTGACTCCACAAGAACTTGATCATATT-3' (mdr 2)

Genomic DNA was isolated from tail biopsies of TGGQ transgenic mice (8). Total RNA was isolated from the thyroid glands of several mice in each 


\section{Short Technical Reports}

line ( $f_{2}$ generation) by the guanidinium isothiocyanate method (2).

A plasmid containing the murine Q209L cDNA was the generous gift of Dr. R. Iyengar (Mt. Sinai Hospital, New York). Plasmid DNA was mixed with mouse genomic DNA at ratios ranging from 0.1 copies per genome to 10 copies per diploid genome (corresponding to mutant to wild-type molar ratios of $0.05-5.0$ ).

\section{RT and PCR}

Reverse transcription was performed in a final volume of $10 \mu \mathrm{L}$ using $1 \mu \mathrm{g}$ of total RNA, 75 pmol of random hexamer primers and $200 \mathrm{U}$ of Maloney murine leukemia virus reverse transcriptase (MMLV-RT) (SUPERSCRIPT $^{\mathrm{TM}}$ RNase $\mathrm{H}^{-}$; Life Technologies, Gaithersburg, MD, USA) according to the manufacturer's recommendations. After cooling to room temperature, $2 \mathrm{U}$ RNase H (Life Technologies) were added. The sample was incubated at $37^{\circ} \mathrm{C}$ for $20 \mathrm{~min}$ and stored at $4^{\circ} \mathrm{C}$.

PCR was performed using Taq I DNA Polymerase (Perkin-Elmer, Norwalk, CT, USA) and primers mdr 1 and mdr $2(0.5 \mu \mathrm{M}$ each) to amplify either genomic DNA (500 ng) or cDNA (10\%). A programmable thermal cycler (MJ Research, Watertown, MA, USA) was used to perform 40 cycles of PCR, each cycle consisting of denaturation $\left(94^{\circ} \mathrm{C}, 1 \mathrm{~min}\right)$, annealing $\left(62^{\circ} \mathrm{C}, 1 \mathrm{~min}\right)$ and extension $\left(72^{\circ} \mathrm{C}, 1 \mathrm{~min}\right)$. In the initial cycle, denaturation was increased to $5 \mathrm{~min}$, and in the final cycle, extension time was increased to $5 \mathrm{~min}$. Ten microliters of the PCR mixture were electrophoresed through an $8 \%$ polyacrylamide gel, and DNA was visualized by ethidium bromide staining and UV transillumination.

For some PCRs, the sense primer (mdr 1) was end-labeled with ${ }^{32} \mathrm{P}$ [dATP] (Amersham, Arlington Heights, IL, USA) using T4 Polynucleotide Kinase (Life Technologies).

\section{DGGE}

Optimal resolution of the PCR products by DGGE was obtained by electrophoresis through $10 \%$ polyacrylamide gels containing a linearly increasing concentration $(40 \%-70 \%)$ of denatu-

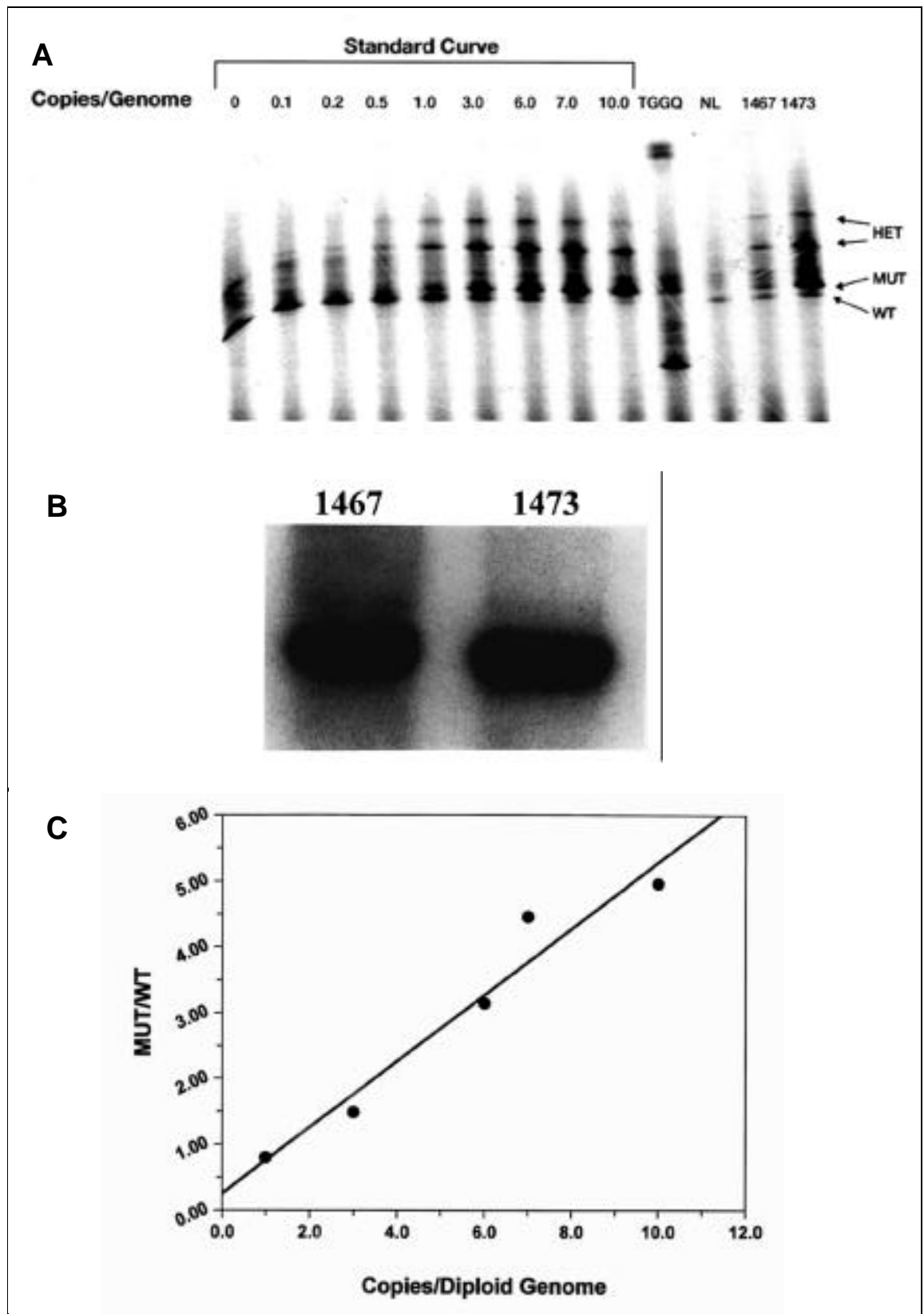

Figure 1. Quantitative DGGE of PCR-amplified $G_{\alpha q}$ fragment from mouse genomic DNA. (A) DGGE analysis of PCR-amplified products. MUT identifies the murine Q209L homoduplex, WT identifies the wild-type murine $\mathrm{G}_{\alpha \mathrm{q}}$ homoduplex. The standard curve lanes represent the addition of increasing copies of Q209L plasmid to normal murine genomic DNA. Lanes 1467 and 1473 are DNA-amplified from two transgenic mice. Positive control Q209L mutant is DNA-amplified from a rat FRTL-5 cell line stably transfected with murine Q209L plasmid (TGGQ), the more rapidly migrating homoduplex band is the wild-type rat $\mathrm{G}_{\alpha \mathrm{q}}$ homoduplex, while the more slowly migrating band represents murine Q209L. The wild-type murine $\mathrm{G}_{\alpha \mathrm{q}}$ control is DNA-amplified from normal mouse (NL). The upper mutant homoduplex becomes more intense relative to the lower wild-type homoduplex as more copies of the mutant DNA are added. Note the early appearance of the two less intense, heteroduplex bands (HET). The two transgenic lines contain different amounts of mutant product relative to the two wild-type copies in the normal mouse genome. (B) Southern blot of genomic DNA extracted from tail biopsies of founder mice from the two transgenic clones included in the DGGE, 1467 and 1473. Densitometric quantification of the bands reveals that genomic DNA isolated from 1473 contained 3.7 times more transgene DNA than 1467. This relationship is consistent with the quantified DGGE results shown in Panel C. (C) The DGGE was quantified by phosphor imaging as described. The y-axis depicts the ratio of mutant to wild-type band radioactivity, and the $\mathrm{x}$-axis depicts the corresponding amount of Q209L plasmid added to the normal mouse genomic DNA before PCR amplification. The 1467 and 1473 mice correspond to the incorporation of 2 and 8 copies of Q209L per mouse diploid genome (MUT/WT ratios of 1:1 and 4:1). 
rants, where $100 \%$ denaturant consists of $8 \mathrm{M}$ urea and $100 \%$ formamide $(1,4)$. Gels were run at $100 \mathrm{~V}$ for $14 \mathrm{~h}$ at $60^{\circ} \mathrm{C}$ in TAE buffer (40 mM Tris- $\mathrm{HCl}, 20$ $\mathrm{mM}$ sodium acetate, $1 \mathrm{mM}$ EDTA, $\mathrm{pH}$ 7.4) as previously described (7).

DNA was visualized by ethidium bromide staining and UV transillumination. When a radiolabeled primer was utilized, the gels were dried and DNA was visualized and quantified using a PhosphorImager ${ }^{\circledR}$ and ImageQuant ${ }^{\circledR}$ software (Molecular Dynamics, Image Quant, Sunnyvale CA, USA).

\section{Southern Blot Analysis}

Genomic DNA was extracted from mouse tail DNA preparations, digested with restriction enzymes BamHI and EcoRI, transferred to nylon membranes and probed with the transgene-specific, 32P-labeled 3' untranslated regions of the $T G G Q$ gene as previously described for a similar transgene using the rat thyroglobulin gene promoter to target the cholera toxin A1 subunit (11). Following high-stringency washing, the membranes were autoradiographed overnight at $-70^{\circ} \mathrm{C}$, and DNA was quantified.

\section{RESULTS}

\section{Transgene Copy Number}

Murine genomic DNA was mixed with Q209L plasmid DNA at ratios corresponding to $0-10$ copies of transgene per haploid mouse genome. Genomic DNA isolated from a rat thyroid cell line (FRTL-5) that had been stably transfected with TGGQ and genomic DNA from normal mice were used as positive and negative controls, respectively. Figure 1 shows PCR products resolved by DGGE (Figure 1A). DGGE resolves the Q209L mutant homoduplex (MUT) and the wild-type mouse $\mathrm{G}_{\alpha \mathrm{q}}$ homoduplex (WT). With increasing copies of Q209L in the PCR mixture, the intensity of the Q209L homoduplex band becomes greater relative to the wild-type band. Quantification of the homoduplex bands by autoradiography revealed a linear increase in the relative intensity of the mutant homoduplex as the concentration of Q209L
DNA was increased (Figure 1C). The more slowly migrating heteroduplex bands (HET) are visible even when very low numbers of Q209L are added, which demonstrates the sensitivity of the DGGE technique.

DNA samples from transgenic mice 1467 and 1473 reveal mutant:wild-type molar ratios of $1: 1$ and $4: 1$, corresponding to 2 and 8 copies of transgene integrated per diploid mouse genome, respectively.

\section{Transgene mRNA Expression}

To compare the levels of Q209L mRNA and the wild-type $G_{\alpha q}$ mRNA expressed in the thyroids of transgenic mice, first-strand cDNA was synthesized from total RNA isolated from pooled thyroids of the $f_{2}$ generation of two affected mouse lines (1467 and 1438). The cDNA was amplified by PCR, and the products were analyzed by DGGE (Figure 2). The level of Q209L mRNA was greatest in the mice containing the most copies of the TGGQ transgene (i.e., 1467) and was associated with markedly increased phospholipase $\mathrm{C}$ activity and the greatest histological changes (8).

\section{Southern Blot Analysis}

Southern blot analysis of the founder mice for the 1467 and 1473 mice are depicted in Figure 1B. The amount of transgene in the 1473 mouse was 3.7 times greater than in the 1467 mouse and was similar to the ratio determined by DGGE. Absolute quantification was attempted, but a linear standard curve was not attained.

\section{DISCUSSION}

Transgenic mice and cell lines are commonly developed as models of human disease. Phenotypic variability in the transgenic animals is due, in part, to differences in both the copy number and the expression of the transgene. Accordingly, accurate determination of transgene copy number and expression is necessary to interpret phenotypic changes in these animals. Currently, transgene copy number is most commonly estimated by analyzing the 


\section{Short Technical Reports}

hybridization intensities of transgenespecific probes to genomic DNA by Southern blot analysis.

We have described a novel PCRbased method for determining transgene copy number that is based on competitive PCR between the endogenous wild-type gene and the mutant transgene. In contrast to standard competitive PCR in which products of different lengths are generated, these PCR products have identical sequences other than a single base pair mutation. Thus, the amplified PCR products can be resolved and quantified under non-denaturing conditions only if a restriction site has been created or lost in the mutant (5). DGGE represents an alternative method for quantitation of identically sized products. The relative intensity of the two homoduplex bands is used to calculate the stoichiometry of the two templates in the PCR. Using this technique, one can detect the integration of a single copy of transgene and is accurate to a copy number of at least ten. The kinetics of PCR make the accurate determination of larger copy numbers by PCR-DGGE problematic; however, the technique is rapid, precise, reproducible and can be performed semiquantitatively after autoradiography or ethidium bromide staining.

DGGE can also be applied to determine transgene expression, as the stoichiometry of transgene genomic mRNAs is resolved by DGGE analysis of RT-PCR products. Determining expression levels of the mutant transgene relative to endogenous wild-type is particularly important in understanding transgene effects, particularly since the number of copies expressed may not correlate with the genomic copy number due to the effects of integration site. In the example shown here, the level of expression of the Q209L RNA correlated with histological changes in the thyroid glands of the transgenic mice and with $\mathrm{PIP}_{2}$ hydrolysis, a measure of $\mathrm{G}_{\alpha q^{-}}$ dependent phospholipase $\mathrm{C}$ activity (8).

In general, the melting point of a particular DNA fragment can be determined by computer analysis (4), perpendicular DGGE, or by resolving PCR products by DGGE through broad denaturing gradients and progressively decreasing the gradient until maximal resolution is attained. PCR product length

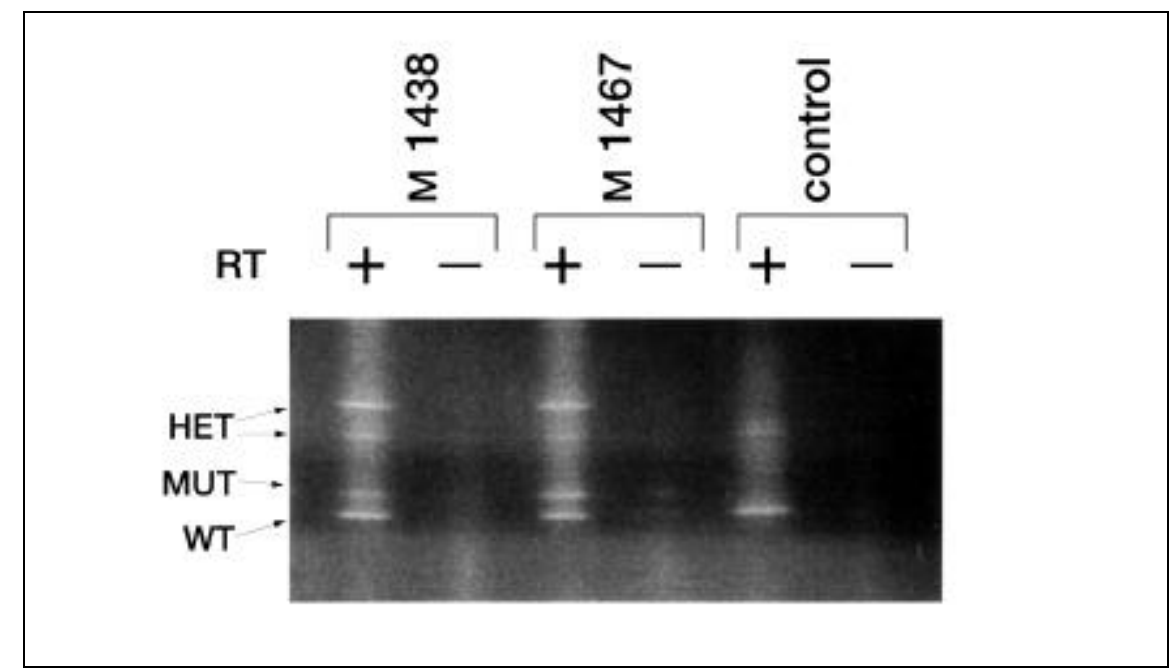

Figure 2. Semiquantitative analysis of expression of Q209L transgene by RT-PCR and DGGE. RTPCR products derived from 2 transgenic mouse lines (1438 and 1467) and one normal mouse (control) evaluated by DGGE to determine relative expression of the transgene. RT negative controls (labeled -) were included for each RNA sample. The upper, mutant (MUT) homoduplex, the lower wild-type (WT) homoduplex and the heteroduplexes (HET) are again seen. The relative expression of the mutant homoduplex correlated with the transgene copy number, activity and histologic alterations in the transgenic mice (8).

should be between 100 and $800 \mathrm{bp}$, and the PCR should be optimized to minimize nonspecific amplification to avoid nonspecific bands on the DGGE (6).

In summary, resolving PCR and RTPCR products by DGGE allows for semiquantitative determinations of copy number and expression of transgenes that differ from genomic genes by only a single nucleotide. Compared to currently utilized methods, semiquantitative DGGE is a rapid, simple and reproducible technique that allows for a more detailed understanding of transgene effects.

\section{REFERENCES}

1.Abrams, E.S., S.E. Murdaugh and L.S. Lerman. 1990. Comprehensive detection of single base changes in human genomic DNA using denaturing gradient gel electrophoresis and a GC clamp. Genomics 7:463-475.

2.Chomczynski, P. and N. Sacchi. 1987. Single-step method of RNA isolation by acid guanidinium thiocyanate-phenol-chloroform extraction. Anal. Biochem. 174:485-488.

3.De Vivo, M., J. Chen, J. Codina and R. Iyengar. 1992. Enhanced phospholipase C stimulation and transformation of NIH-3T3 cells expressing Q209L Gq-alpha-subunits. J. Biol. Chem. 267:18263-18266.

4.Fischer, S.G. and L.S. Lerman. 1983. Separation of random fragments of DNA according to properties of their sequences. Proc. Natl. Acad. Sci. USA 80:1579-1583.

5.Gilliland, G., S. Perrin and H.F. Bunn.
1990. Competitive PCR for quantitation of mRNA, p. 60-69. In M.A. Innis, D.H. Gelfand, J.J. Sninsky and T.J. White (Eds.), PCR Protocols. A Guide to Methods and Applications. Academic Press, San Diego.

6.Hovig, E., B. Smith-Sorenson and A-L. Borresen. 1995. Detection of mutations by denaturing gradient gel electrophoresis, p. 7.5.17.5.12. In N.C. Dracopoly, J.L. Haines, B.R. Korf, D.T. Moir, C.C. Morton, C.E. Seidman, J.G. Seidman and D.R. Smith (Eds.), Current Protocols in Human Genetics. John Wiley \& Sons, New York.

7.Miric, A. and M.A. Levine. 1992. Analysis of the preproPTH gene by denaturing gradient gel electrophoresis in familial isolated hypoparathyroidism. J. Clin. Endocrinol. Metab. 74:509-516.

8.Saji, M., Y. Takiyama, W.H. Westra, M.D. Ringel, L.D. Kohn, M.A. Levine and M.A. Zeiger. 1996. Thyroid-specific expression of activated $\mathrm{G}_{\mathrm{q} \alpha}$ Q209L causes thyroid hyperplasia in transgenic mice. Thyroid. 6 (Supp. 1):S65.

9.Schwindinger, W.F., C.A. Francomano and M.A. Levine. 1992. Identification of a mutation in the gene encoding the $\alpha$ subunit of the stimulatory $\mathrm{G}$ protein of adenylyl cyclase in McCune-Albright syndrome. Proc. Natl. Acad. Sci. USA 89:5152-5156.

10.Strathman, M. and M.I. Simon. 1990. G Protein diversity: a distinct class of $\alpha$ subunits is present in vertebrates and invertebrates. Proc. Natl. Acad. Sci. USA 87:9113-9117.

11.Zeiger, M.A., M. Saji, Y. Gusev, W.H. Westra, Y. Takiyama, W.C. Dooley, L.D. Kohn and M.A. Levine. 1997. Thyroid-specific expression of cholera toxin A1 subunit causes thyroid hyperplasia and hyperthyroidism in transgenic mice. Endocrinology 138:31333140 . 
Supported in part by research grants from the United States Public Health Service R01-DK-34281 (M.A.L.), a Clinical Associate Physician Award (RR-007222251) from the National Institutes of Health (W.F.S.), NIH, NCCR, OPD-GCRC Grant No. RR00052 and a grant from the Interthy Research Foundation (MS). This research was performed while Dr. Ringel was a Pfizer Postdoctoral Fellow. Address correspondence to Dr. Michael A. Levine, The Johns Hopkins University School of Medicine, 720 Rutland Street, 863 Ross Building, Baltimore, MD 21205, USA. Internet: mlevine@ welchlink.welch.jhu.edu

Received 10 March 1997; accepted 29 July 1997.

M.D. Ringel, W.F. Schwindinger, M. Saji, M.A. Zeiger and M.A. Levine Johns Hopkins University School of Medicine Baltimore, MD, USA

\section{Adaptation of Standard Spreadsheet Software for the Analysis of DNA Sequences}

BioTechniques 23:131-138 (January 1998)

\begin{abstract}
This paper presents use of spreadsheet software to derive various statistics on nucleotide and codon distribution and frequency from gene sequence data, including $\chi^{2}$ tests and their derivatives, and proportion of nucleotides in the third position. The basic principles can be easily extended to other more complex functions. In addition, it can be used to translate a nucleic acid sequence into a protein sequence or to produce a codon usage table. This adaptation permits sequence analysis without expensive dedicated software and easy modification of the analysis according to the user's requirements. It is also ideal for introducing biological science students to the programming potential of spreadsheets.
\end{abstract}

\section{INTRODUCTION}

The ever increasing number of DNA sequences being deposited in various databases necessitates the easy availability of simple computer programs for sequence analysis. Frequently, a problem arises in finding a program able to perform the exact type of analysis required by the experimenter. To most molecular biologists, untrained in computer programming languages, the prospect of having to write a new program or even alter an existing one is a daunting task. A solution to this problem may lie in use of the common spreadsheet program such as Microsoft $^{\circledR}$ Excel $^{\circledR}$, as suggested by Shaw (9), whose original paper adapted Excel for the analysis of protein sequences. Here, we present an Excel adaptation that can be used for the analysis of DNA sequences, generating a range of statistics on nucleotide and codon usage, in addition to performing translation. Further modification of the program to perform more complex functions requires only a familiarity with Excel.

\section{MATERIALS AND METHODS}

\section{Computer Programs Used}

The programs used in this work are those found already installed in a wide range of $\mathrm{IBM}^{\circledR}$-compatible personal computers-Netscape to allow access to the Internet, Microsoft Word ${ }^{\mathrm{TM}}$ Version 6.0 (Microsoft, Redmond, WA, USA) as a word-processing program and Microsoft Excel Version 5.0 as a spreadsheet program. However, other Internet viewers, spreadsheet packages and word-processing documents can be used instead.

\section{Source of DNA Sequences}

DNA sequences for input can be accessed and downloaded directly from databases available on the Internet or can be copied directly from sequence information generated by an automated sequencer. DNA sequence information for testing the program was accessed from the GenBank ${ }^{\circledR}$ database (http: //www2.ncbi.nlm.nih.gov/cgi-bin/ genbank). Sequences from GenBank 